\title{
Optimal Geometric Disks Covering using Tessellable Regular Polygons
}

\author{
Elvis K. Donkoh ${ }^{1}$, Alex A. Opoku ${ }^{2}$ \\ ${ }^{1,2}$ School of Sciences, Department of Mathematics and Statistics, University of Energy \& Natural Resources, Sunyani, \\ Ghana. \\ Correspondence: Elvis K. Donkoh, School of Sciences, Department of Mathematics and Statistics, University of Energy \\ \& Natural Resources, Sunyani, Ghana, Box 214, Sunyani, Ghana. Tel: +233507452075. E-mail: \\ elvis.donkor@uenr.edu.gh.
}

Received: January 22, 2016

Accepted: February 15, 2016 Online Published: March 9, 2016

doi:10.5539/jmr.v8n2p25

URL: http://dx.doi.org/10.5539/jmr.v8n2p25

\begin{abstract}
Geometric Disks Covering (GDC) is one of the most typical and well studied problems in computational geometry. Geometric disks are well known 2-D objects which have surface area with circular boundaries but differ from polygons whose surfaces area are bounded by straight line segments. Unlike polygons covering with disks is a rigorous task because of the circular boundaries that do not tessellate. In this paper, we investigate an area approximate polygon to disks that facilitate tiling as a guide to disks covering with least overlap difference. Our study uses geometry of tessellable regular polygons to show that hexagonal tiling is the most efficient way to tessellate the plane in terms of the total perimeter per area coverage.
\end{abstract}

Keywords: Disks, geometric covering, hexagon, polygons, tiling.

\section{Introduction}

Tiling differs from covering in that the former is a family of sets without overlap whereas the latter covers the entire plane with no gaps but with overlaps (Lessard, 2000, p.17). Triangles, squares and hexagons are known to be the only Archimedean tiling's with lattice polygon (Ding, 2010, p.7). Any regular polygon that can tile has the property of covering. It is often useful to consider the single regular polygon whose area approximates that of a circle. This regular polygon could be a guide in our geometric disks covering problem.

\section{Related Literature}

Covering has been one of the most fundamental and yet challenging issues in wireless network and found many applications such as routing and broad casting (Xu and Whang 2011, pp. 108-118). A natural dual to covering is the corresponding tilling. Tiling $T$ is a countable family of closed sets $\left\{T_{1}, T_{2}, T_{3}, \ldots T_{n}\right\}$ which covers the Euclidean plane without any gaps or overlaps, (Keating and King, 1999, pp. 83-91). Here $T_{1}, T_{2}, T_{3}, \ldots$ are known as the tiles of $T$. When the set of polygons has the same shape and size then it is a monohedral tiling. The only edge-to-edge monohedral tiling's by regular polygons are tiling of squares, equilateral triangles and regular hexagons (Lessard, 2000, p. 17).

Sirbu (1992, pp. 174-178), has shown that plane tiling's and their properties have applications in medicine, where tiling's are used to describe the fight between the immune system and a pathogen agent.

Paredes et.al (1998, pp.11990-11995), stated that tiling with squares and triangles are very useful tools to study several structural and thermodynamical properties of a wide variety of solids.

Keating and King (1999, pp. 83-91) investigated a necessary and sufficient condition for a bounded region of the plane with rectangles to be tillable with finitely many squares.

Melissen and Schuur (1996). studied how to cover a bounded fixed square with a small number of circles by expressing the relationship between the radius of the circle and the side of the square. But our study focuses on disks covering using hexagons and proves that hexagonal tiling is the best approach to our approximate disks covering problem.

\section{Computational Experience}

\subsection{Tessellable Regular Polygon}

Among all the tessellable regular polygons viz equilateral triangle, squares, etc hexagon has the closest area to that of a disk.

Theorem 1: The area of a regular hexagon is closer to its circumcircle than to any tessellable regular polygon (like a 
square, equilateral triangle) to that of its circumcircle.

Proof

Consider the circle with radius $R_{1}$ inscribed in a hexagon, the other by a square and the third by a triangle as shown in Figure 1

Case I: Hexagon $A B C D E F$

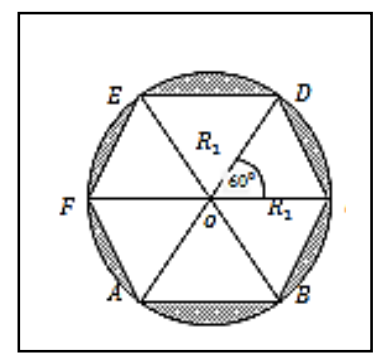

Figure 1 (a). Hexagon inscribed in a circle

Each triangle in Figure 1 (a) is equilateral so we have $\angle D O C=\frac{\pi}{3}$.

$$
\begin{gathered}
\text { Area of } \triangle D O C=\frac{1}{2} \times|O D \| O C| \sin \frac{\pi}{3} \\
=\frac{R_{1}^{2} \sqrt{3}}{4} \text { square units }
\end{gathered}
$$

$\therefore$ Area of all six equilateral triangles $=6 \times$ area of $\triangle D O C$

Case II: Square $A B C D$

$$
\begin{aligned}
& =6 \times \frac{R_{1}^{2} \sqrt{3}}{4} \\
& \cong 2.598 R_{1}^{2} \text { square units. }
\end{aligned}
$$

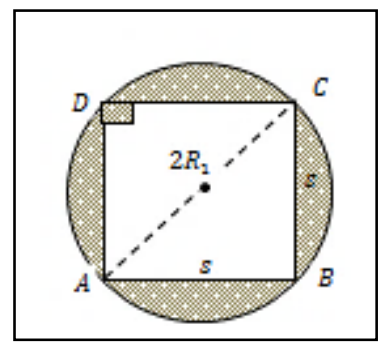

Figure 1 (b). Square inscribed in a circle

We consider the isosceles right triangle $A B C$ and apply Pythagoras theorem:

$$
\begin{aligned}
& \left(2 R_{1}\right)^{2}=s^{2}+s^{2} \\
& \Rightarrow s=R_{1} \sqrt{2} \text { units. }
\end{aligned}
$$

But area of square $A B C D=|A B| \times|B C|$

$$
\begin{aligned}
& =s \times s \\
& =2 R_{1}^{2} \text { square units. }
\end{aligned}
$$

Case III: Equilateral triangle $A B C$

Consider the equilateral triangle $A B C$ inscribed in a circle with centre $O$, radius $r$ and side $s$. 


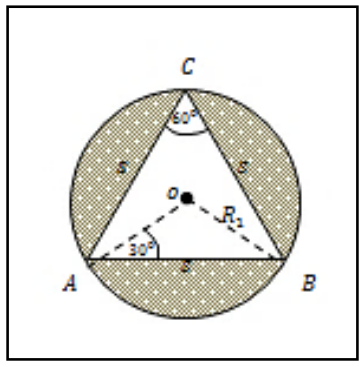

(i)

Equilateral triangle $A B C$

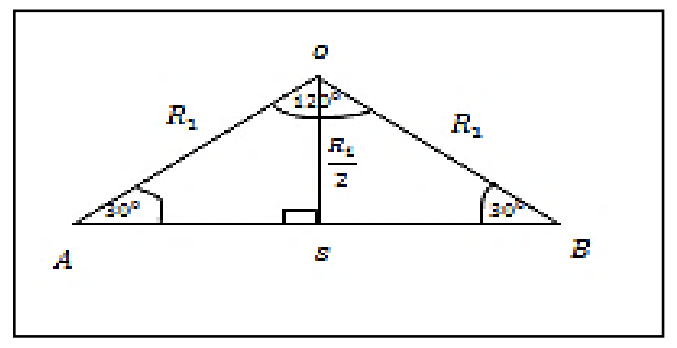

(ii) Isosceles triangle $A B O$

Figure 1(c). Triangle inscribed in a circle

$$
\begin{aligned}
& \text { Area of } \triangle A B C=\sqrt{s(s-a)(s-b)(s-c)} \text { where } \\
& s=\frac{a+b+c}{2} \quad \text { is the semi-perimeter } \\
& =\frac{s+s+s}{2} \\
& =\frac{3 s}{2} \\
& \text { Area of } \triangle A B C=\sqrt{\frac{3 s}{2}\left(\frac{3 s}{2}-s\right)\left(\frac{3 s}{2}-s\right)\left(\frac{3 s}{2}-s\right)} \\
& =\sqrt{\frac{3 s^{4}}{16}} \\
& \text { Area of } \triangle A B C=0.4330 \mathrm{~s}^{2}
\end{aligned}
$$

But in Figure $3.1(c)(i i)$ we can find $s=f(r)$

$$
\begin{aligned}
\cos \left(\frac{\pi}{6}\right) & =\frac{s / 2}{R_{1}} \\
s & =R_{1} \sqrt{3} \\
\therefore \text { area of } \triangle A B C & =0.4330 \times R_{1}^{2} \times 3 \\
& =1.299 \mathrm{R}_{1}{ }^{2}
\end{aligned}
$$

Case IV: Circle $A B C D E F$

$$
\begin{aligned}
\text { Area } & =\pi \times R_{1}^{2} \\
& \cong 3.142 R_{1}^{2^{2}} \text { to } 3 \mathrm{~d} . \mathrm{p}
\end{aligned}
$$

The common area of circles based on three polygons and occupying ratio in a circle has been shown in Table.1.

Table 1. Occupying Ratio Comparison of Tessellable Lattice Polygon.

\begin{tabular}{lccc}
\hline Shape & Triangle & Square & Hexagon \\
\hline Area of polygon $(A)$ & $1.299 R_{1}^{2}$ & $2 R_{1}^{2}$ & $2.598 R_{1}^{2}$ \\
\hline Area of circle $(B)$ & $3.142 R_{1}^{2}$ & $3.142 R_{1}^{2}$ & $3.142 R_{1}^{2}$ \\
\hline Ratio $(A: B)$ & $41.34 \%$ & $63.65 \%$ & $82.69 \%$
\end{tabular}

Comparing the areas obtained for the three geometrical shapes we conclude that hexagons approximate circles more closely than squares, regular triangles and generally than any other regular tessellable geometrical 2-dimensional polygon. 
Corollary: Hexagons, because they approximate circles more closely are more compact than squares. This fact has direct application to any point of set sensors arranged on a plane or similar surface and can be reflected in nature (e.g. most animal vision organs have rods and cones arranged in nearly hexagonal tessellations in the eyes fovea), (Raposo , 2011, pp. 37).

\subsection{Non-Tessellable Regular Polygon}

Let $A_{n}$ be the area of the inscribed polygon with $n$ sides. As $n$ increases, it appears that $A_{n}$ becomes closer and closer to the area of the circle. We say that the area of the circle is the limit of the areas of the inscribed polygons, and we write

$$
A=\lim _{n \rightarrow \infty} A_{n}
$$

Theorem 2: The area of an $n$ sided non-tessellable regular polygon inscribed in a circle is closer to its circumcircle as $n$ increases.

Proof:

Non-lattice Archimedean non-tillable regular polygon includes pentagon, heptagon, octagon, nonagon, decagon, etc. We shall give a proof that as the number of sides of a non-tessellable regular polygon increases its area approximates that of it circumcircle than any regular tessellable polygon. We shall give the percentage occupying area proof using geometry by considering the following cases.

Case I: Pentagon $A B C D E$

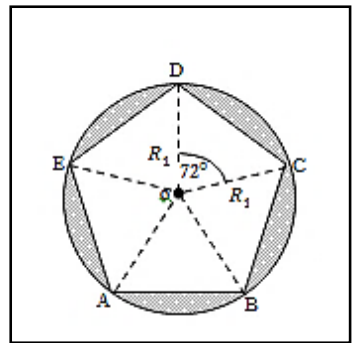

Figure 2. Pentagon inscribed in a circle

It is evident that each of the triangle in Figure 2 is isosceles. So we consider $\triangle D O C$ where characteristically $\angle D O C=$ $\frac{\pi}{5}$

$$
\text { Area of } \begin{aligned}
\triangle D O C & =\frac{1}{2} \times|O D \| O C| \sin \left(\frac{\pi}{5}\right) \\
& =0.4756 R_{1}^{2}
\end{aligned}
$$

$\therefore$ Area of all five isosceles triangles

\section{Case II: Heptagon $A B C D E F G$}

$$
\begin{aligned}
& =5 \times \text { area of } \triangle D O C \\
& =5 \times 0.4756 R_{1}^{2} \\
& \cong 2.3780 R_{1}^{2}
\end{aligned}
$$

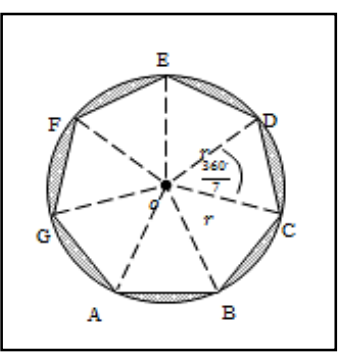

Figure 3. Heptagon inscribed in a circle 


$$
\begin{aligned}
\text { Area of } \triangle D O C= & \frac{1}{2} \times|O D \| O C| \sin \left(\frac{2 \pi}{7}\right) \\
& =\frac{1}{2} \times r \times r \times 0.7818 \\
& =0.3909 R_{1}^{2} \\
& =7 \times \text { area of } \triangle D O C \\
& =7 \times 0.3909 R_{1}^{2} \\
& \cong 2.7363 R_{1}^{2}
\end{aligned}
$$

Case III: Octagon $A B C D E F G H$

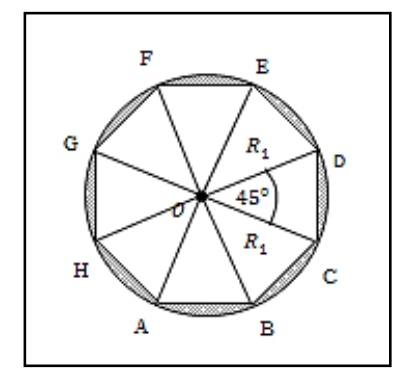

Figure 4. Octagon inscribed in a circle

$$
\begin{aligned}
\text { Area of } \triangle D O C & =\frac{1}{2} \times|O D||O C| \sin \left(\frac{\pi}{4}\right) \\
& =\frac{\sqrt{2}}{4} R_{1}^{2} \\
\therefore \text { Area of all octagon } & =8 \times \text { area of } \triangle D O C \\
& =8 \times \frac{\sqrt{2}}{4} R_{1}^{2} \\
& \cong 2.8284 R_{1}^{2}
\end{aligned}
$$

Case IV: $n$ sided polygon

Generally, area of polygon inscribed in a circle with sides $n$ is

$$
\begin{aligned}
A_{n}= & n \times(\text { area of a single triangle with origin as one vertex }) \\
& =n \times \frac{1}{2} \times R_{1} \times R_{1} \times \sin \left(\frac{2 \pi}{n}\right) \\
A_{n} & =\frac{n R_{1}^{2}}{2} \sin \left(\frac{2 \pi}{n}\right)
\end{aligned}
$$

Case V: Circle

$$
\begin{aligned}
\text { Area } & =\pi \times R_{1}^{2} \\
& \cong 3.142 R_{1}^{2} \text { to } 3 \text { decimal places. }
\end{aligned}
$$

The common area of circles based on some non-tessallable regular polygons and occupying ratio in a circle has been shown in Table 2. 
Table 2. Occupying Ratio Comparison of non-tilling Archimedean shapes.

\begin{tabular}{lcclc}
\hline Non-tilling Shape & Pentagon & Heptagon & Octagon & $n$-sided polygon \\
\hline Area $(A)$ & $2.3780 R_{1}^{2}$ & $2.7363 R_{1}^{2}$ & $2.8284 R_{1}^{2}$ & $\frac{n R_{1}^{2}}{2} \times \sin \left(\frac{2 \pi}{n}\right)$ \\
\hline Area of $\operatorname{circle}(B)$ & $3.142 R_{1}^{2}$ & $3.142 R_{1}^{2}$ & $3.142 R_{1}^{2}$ & $3.142 r^{2}$ \\
\hline Ratio $(A: B)$ & $75.68 \%$ & $87.71 \%$ & $90.02 \%$ & $\frac{n}{2 \pi} \times \sin \left(\frac{2 \pi}{n}\right) \%$
\end{tabular}

Table 2 shows the area on an $n$ sided non-tessellable regular polygon approaching the area of a circle as $n$ increases. We shall state a theorem to that effect and give the first formal analytical proof.

Theorem 3: Recall $A_{n}$ as in theorem (2). Then

$$
\lim _{n \rightarrow \infty} A_{n}=\pi R_{1}^{2}
$$

Remark: The above theorem states that the area $A_{n}$ of an $n$-sided regular non-tessellable polygon inscribed in a circle with radius $R_{1}$ approximates the area $\pi R_{1}^{2}$ of the circle, as $n$ becomes large.

Proof.

We shall give the first analytical proof.

From equation (2) $\quad A_{n}=\frac{n R_{1}^{2}}{2} \sin \left(\frac{2 \pi}{n}\right)$

$$
\begin{aligned}
\lim _{n \rightarrow \infty} A_{n} & =\lim _{n \rightarrow \infty} \frac{n R_{1}^{2}}{2} \sin \left(\frac{2 \pi}{n}\right) \\
& =\frac{R_{1}^{2}}{2} \lim _{n \rightarrow \infty} n \times \sin \left(\frac{2 \pi}{n}\right) \\
& =\frac{R_{1}^{2}}{2} \lim _{n \rightarrow \infty} n \times \sin \left(\frac{2 \pi}{n}\right) \times \frac{2 \pi / n}{2 \pi / n} \\
& =\frac{R_{1}^{2}}{2} \lim _{n \rightarrow \infty} n \times 2 \pi / n \times \frac{\sin \left(\frac{2 \pi}{n}\right)}{2 \pi / n} \\
& =\frac{2 \pi R_{1}^{2}}{2} \times \lim _{n \rightarrow \infty} n \times \frac{1}{n} \times \frac{\sin \left(\frac{2 \pi}{n}\right)}{2 \pi / n} \\
& =\pi R_{1}^{2} \times \lim _{n \rightarrow \infty} \frac{\sin \left(\frac{2 \pi}{n}\right)}{2 \pi / n} \\
\lim _{n \rightarrow \infty} A & =\pi R_{1}^{2} \quad\left(\mathrm{~L}^{\prime} \operatorname{Hopital} \mathrm{s} \mathrm{rule,} \mathrm{indeterminate} \quad \text { type } \frac{0}{0} \quad \text { case }\right) \\
& =\pi R_{1}^{2} \times \lim _{n \rightarrow \infty} \frac{D_{n}\left[\sin \left(\frac{2 \pi}{n}\right)\right]}{D_{n}\left(\frac{2 \pi}{n}\right)} \\
& =\pi R_{1}^{2} \times \lim _{n \rightarrow \infty} \frac{\left(\frac{-2 \pi}{n^{2}}\right) \cos \left(\frac{2 \pi}{n}\right)}{\left(\frac{-2 \pi}{n^{2}}\right)} \\
& =\pi R_{1}^{2} \times \lim _{n \rightarrow \infty} \cos \left(\frac{2 \pi}{n}\right) \times 1 \\
& =\pi R_{1}^{2} \times \cos 0 \times 1 \\
\lim _{n \rightarrow \infty} A_{n} & =\pi R_{1}^{2}
\end{aligned}
$$

This establishes the fact that the area of a regular polygon with sides $n$ inscribed in a circle of radius $R_{1}$ approaches $\pi R_{1}^{2}$ as $n$ becomes large. Table 4 illustrates the percentage occupying ratio of some non-tessellable regular polygon.

Table 4. Occupying Ratio Comparison of non-tessellable regular polygon. 


\begin{tabular}{lcccc}
\hline Non-tessellable Shape & Pentagon & Heptagon & Octagon & $n$-gon $\rightarrow \infty$ \\
\hline Area of polygon $(A)$ & $2.3780 R_{1}^{2}$ & $2.7363 R_{1}^{2}$ & $2.8284 R_{1}^{2}$ & $\pi R_{1}^{2}$ \\
\hline Area of circle $(B)$ & $\pi R_{1}^{2}$ & $\pi R_{1}^{2}$ & $\pi R_{1}^{2}$ & $\pi R_{1}^{2}$ \\
\hline Ratio $(A: B)$ & $75.68 \%$ & $87.71 \%$ & $90.02 \%$ & $100 \%$ \\
\hline
\end{tabular}

Proposition: For any positive constant $c$, the function $x \mapsto A(x)$ given by

$$
A(x)=\operatorname{cxsin}\left(\frac{2 \pi}{x}\right)
$$

is strictly increasing on $[2, \infty)$.

Proof

Note that $A$ is at least twice continuously differentiable on $\mathbb{R}$ and

$$
\begin{aligned}
A^{\prime}(x) & =c \sin \left(\frac{2 \pi}{x}\right)-\frac{2 \pi c}{x^{2}} \cos \left(\frac{2 \pi}{x}\right) \text { and } \\
A^{\prime \prime}(x) & =-\frac{2 \pi c}{x^{2}} \cos \left(\frac{2 \pi}{x}\right)+\frac{2 \pi c}{x^{2}} \cos \left(\frac{2 \pi}{x}\right)-\frac{4 \pi^{2} c}{x^{3}} \sin \left(\frac{2 \pi}{x}\right) \\
& =-\frac{4 \pi^{2} c}{x^{3}} \sin \left(\frac{2 \pi}{x}\right)
\end{aligned}
$$

Note that

$$
\begin{aligned}
& A^{\prime}(2)=c\left[\sin \left(\frac{2 \pi}{2}\right)-\frac{2 \pi}{4} \cos \left(\frac{2 \pi}{2}\right)\right] \\
& A^{\prime}(2)=\frac{\pi c}{2}>0 \text { for } c>0 \text { and } A^{\prime \prime}(x)<0 \text { for } x \in(2, \infty), \text { therefore } A^{\prime}(x) \text { is strictly decreasing on }
\end{aligned}
$$

$(2, \infty)$. Furthermore $\lim _{x \rightarrow \infty} A^{\prime}(x)=0$. Hence $A^{\prime}(x)$ is strictly positive on $[2, \infty)$, which implies that $A(x)$ is strictly increasing on $[2, \infty)$. The strictly increasing function $A$ indicates that the area of a regular polygon inscribed in a disk increases with respect to the number of sides .i.e $A_{3}<A_{4}<\cdots . A_{n}<A_{n+1}<\cdots$. This is shown in Figure 5.
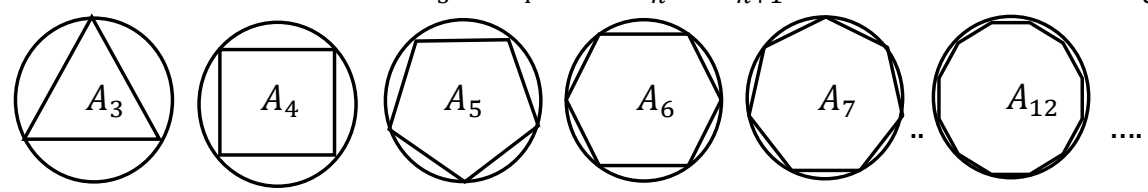

Figure 5. Strictly increasing function $A_{n}(x)$

\subsection{Geometry of Tessellable Polygons with Disks Covering}

Geometrically, we illustrate a plane tiling with equilateral triangle, squares and hexagon. Hexagon is conveniently chosen because it is the only shape that is closest to being circular with the widest area. Figure 6 shows this concepts and the percentage overlap of a segment.

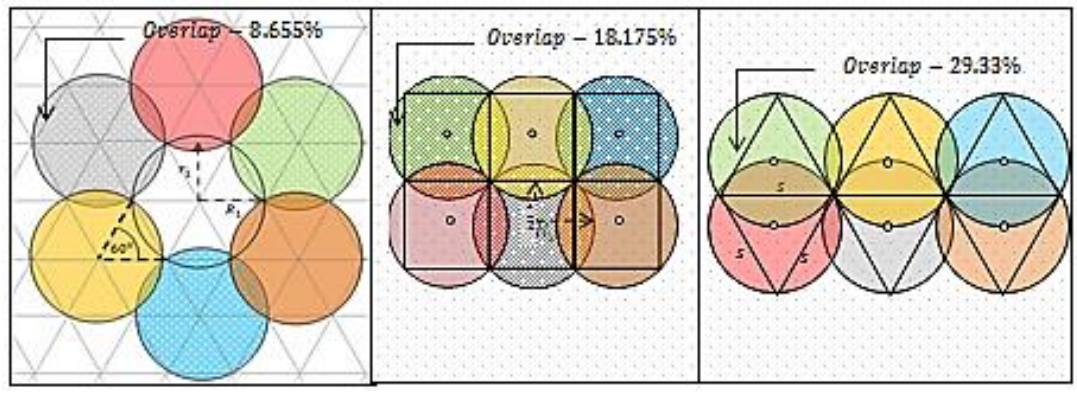
(a) Hexagonal tiling
(b) Square tiling
(c) Equilateral tiling

Figure 6. Tessellable regular polygons with percentage segment overlap 


\subsection{Overlap for Optimal Disks Covering}

Figure 7(a) illustrates the hexagonal cell layout. The inradius and the circumradius of the hexagonal cell are $r_{1}$ and $R_{1}$, respectively. In Figure 7(b), cells are partially overlapped because $R_{1}$ equals to the hexagon's circumradius. The model considers nodes not belonging to the cell of interest. Algebraically the best positioning of the GSM network is where the hexagonal and circular cells overlap to give us a difference of $2\left(R_{1}-r_{1}\right)$ as shown in Figure 7(b).

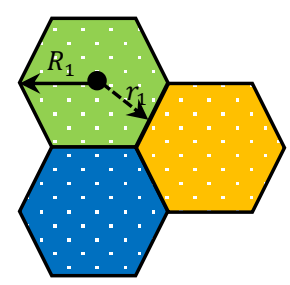

(a) Hexagonal cell layout

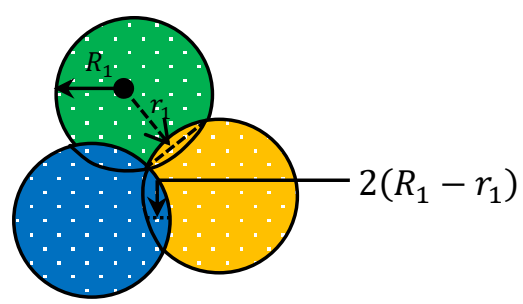

(b) Idealized circular layout

Figure 7. Cell Layout Models For Optimal disks covering.

\subsection{Overlap Difference for Optimal Disks Covering}

We deduce formulas for calculating the width of any hexagonal disks covering in terms of the apothem $\left(r_{1}\right)$ or the radius of the disks $\left(R_{1}\right)$ or the height of the overlap area $(H)$. Consider two intersecting uniform disks shown in Figure 8.

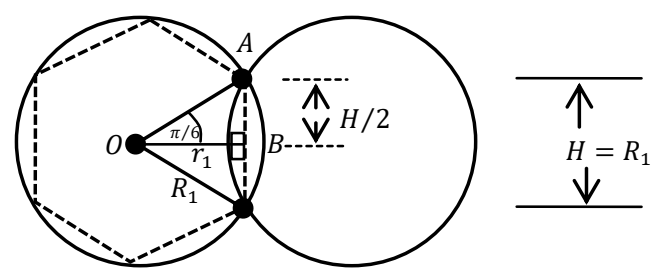

Figure 8. Overlap width for uniform disks

Consider triangle $O A B$ in Figure 8.

Case I:

$$
\sin \left(\frac{\pi}{6}\right)=\frac{A B}{O A}
$$

$$
\frac{1}{2}=\frac{H / 2}{R_{1}} \quad H=R_{1}
$$

Width $=2\left(R_{1}-r_{1}\right)$ or

$$
\text { Width }=2\left(H-r_{1}\right)
$$

Case II:

$$
\begin{aligned}
\operatorname{Cos}\left(\frac{\pi}{6}\right) & =\frac{O B}{O A} \\
\frac{\sqrt{3}}{2} & =\frac{r_{1}}{R_{1}}, \\
r_{1} & =\frac{\sqrt{3}}{2} R_{1} \quad \text { or } \quad r_{1}=\frac{\sqrt{3}}{2} H
\end{aligned}
$$

where $H=R_{1}=\frac{2 r}{\sqrt{3}}=\frac{2 r_{1} \sqrt{3}}{3}$

$$
\begin{aligned}
\text { Width } & =2\left(R_{1}-\frac{\sqrt{3}}{2} R_{1}\right) \\
& =(2-\sqrt{3}) R_{1}=(2-\sqrt{3}) H
\end{aligned}
$$

Case III: $\quad \tan \left(\frac{\pi}{6}\right)=\frac{A B}{O B}, \quad \frac{1}{\sqrt{3}}=\frac{H / 2}{r_{1}}$ 


$$
\begin{aligned}
H & =\frac{2 r_{1}}{\sqrt{3}} \quad \text { or } \quad H=R_{1}=\frac{2 \sqrt{3} r_{1}}{3} \\
\text { Width } & =2\left(\frac{2 \sqrt{3} r_{1}}{3}-r_{1}\right) \\
& =\frac{2}{3}(2 \sqrt{3}-3) r_{1}
\end{aligned}
$$

Equation (5), (6) and (7) establishes the formula for calculating the width of a hexagonal disks covering. Table 5 shows the overlap difference and their percentage occupying ratio.

Table 5. Occupying overlap difference and ratio for uniform disks.

\begin{tabular}{lccc}
\hline \multicolumn{1}{c}{ Regular Polygon } & Hexagon & Square & Equilateral Triangle \\
\hline \multirow{2}{*}{ Overlap Difference $(\boldsymbol{A})$} & $2\left(R_{1}-r_{1}\right)$ & $\frac{2}{3}\left(3 R_{1}-\sqrt{6} r_{1}\right)$ & non \\
\cline { 2 - 4 } & & $(2-\sqrt{2}) R_{1}$ & $R_{1}$ \\
\cline { 2 - 4 } & $\frac{2}{3}(2 \sqrt{3}) R_{1}$ & $\frac{2}{3}(2 \sqrt{3}-\sqrt{6}) r_{1}$ & $\frac{2 \sqrt{3}}{3} r_{1}$ \\
\hline Overlap difference : disks(B) & $2 R_{1}$ & $2 R_{1}$ & $2 R_{1}$ \\
\hline Ratio $(A: B)$ & $13.397 \%$ & $29.289 \%$ & $50 \%$ \\
\hline
\end{tabular}

Theorem 4. The apothem $r_{n}$ created by $n$ sided tessellable regular polygon inscribed in a disk of radius $R_{1}$ is $r_{n}=R_{1}$ os $\left(\frac{\pi}{n}\right)$.

Proof.

Consider Figure 9.

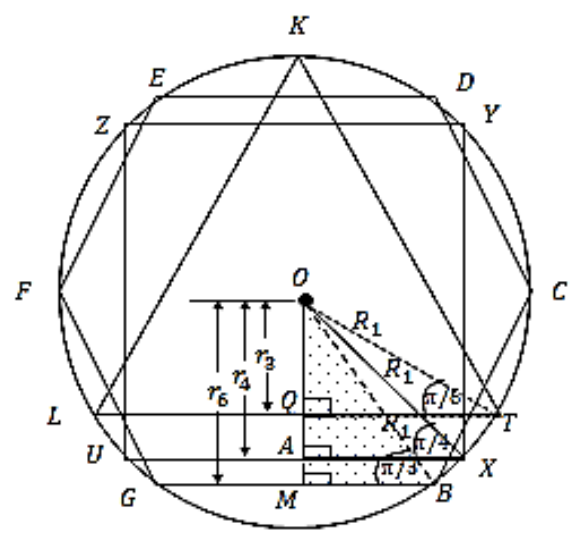

Figure 9. Apothem for regular polygon inscribed in disks

Let the apothem of an $n$ sided regular polygon be $r_{n}$.

Case I: Equilateral triangle $K L T$.

Consider $\triangle Q O T$, in Figure 9. Then

$$
\begin{aligned}
\cos \left(\frac{\pi}{3}\right) & =\frac{r_{3}}{R_{1}} \\
r_{3} & =R_{1} \cos \left(\frac{\pi}{3}\right)
\end{aligned}
$$

Case II: Square $X Y Z U$.

Consider $\triangle A O X$, in Figure 9 then

$$
\cos \left(\frac{\pi}{4}\right)=\frac{r_{4}}{R_{1}}
$$




$$
r_{4}=R_{1} \cos \left(\frac{\pi}{4}\right)
$$

Case III: Hexagon $B C D E F G$

Consider $\triangle M O B$, in Figure 9. Then

$$
\begin{aligned}
& \cos \left(\frac{\pi}{6}\right)=\frac{r_{6}}{R_{1}} \\
& r_{6}=R_{1} \cos \left(\frac{\pi}{6}\right)
\end{aligned}
$$

Generally for an $n$ sided regular polygon,

$$
r_{n}=R_{1} \cos \left(\frac{\pi}{n}\right)
$$

Theorem 5: The total overlap difference created by $n$ sided tessellable regular polygon inscribed in a disk for covering with radius $R_{1}$ is $2 n R_{1}\left[1-\cos \left(\frac{\pi}{n}\right)\right]$. Each overlap difference is $2 R_{1}\left[1-\cos \left(\frac{\pi}{n}\right)\right]$.

Proof.

Let $d_{n}$ denote the overlap difference of an $n$ sided tessellable polygon. We shall prove by induction that when $n \geq 3$ theorem 5 is true.

For $n=3$;

$$
\begin{aligned}
& d_{3}=2 R_{1}\left[1-\cos \left(\frac{\pi}{3}\right)\right] . \\
& d_{3}=R_{1} \quad \text { as shown in Table } 5 .
\end{aligned}
$$

For $n=4$;

$$
\begin{aligned}
& d_{4}=2 R_{1}\left[1-\cos \left(\frac{\pi}{4}\right)\right] . \\
& d_{4}=(2-\sqrt{2}) R_{1} \text { as shown in Table } 5 .
\end{aligned}
$$

For $n=6$;

$$
\begin{aligned}
d_{6} & =2 R_{1}\left[1-\cos \left(\frac{\pi}{6}\right)\right] . \\
& =2 R_{1}\left(1-\frac{\sqrt{3}}{2}\right) \\
d_{6} & =(2-\sqrt{3}) R_{1} \text { as shown in Table } 5 .
\end{aligned}
$$

In telecommunication network design the overlap difference $d_{n}$ help engineers to estimate before hand the overlap cost per choice of tessellable regular polygon. As the overlap difference increase with a decrease in the size of the regular polygon.

Theorem 6: The total overlap area created by $n$ sided tessellable regular polygon inscribed in a disks for covering of radius $R_{1}$ is $\left[\pi-n \sin \left(\frac{2 \pi}{n}\right)\right] R_{1}^{2}$.

Proof.

From Figure 3.8, the area of each overlap difference is

$$
\begin{gathered}
\Delta_{n}=\text { area of disks }-2 \times(\text { area of tessellable regular polygon }) \\
=\pi R_{1}^{2}-2 \times \frac{n R_{1}^{2}}{2} \sin \left(\frac{2 \pi}{n}\right) \\
=\pi R_{1}^{2}-n R_{1}^{2} \sin \left(\frac{2 \pi}{n}\right) \\
\Delta_{n}=\left[\pi-n \sin \left(\frac{2 \pi}{n}\right)\right] R_{1}^{2}
\end{gathered}
$$




\section{Analysis of Results}

Table 1 shows that it is only possible to compute the width of a hexagonal disks covering if either the radius of the disks is known or the apothem of the inscribed hexagon. It is also an establish fact that $R_{1}>r_{1}$ and as $R_{1}$ increases the overlap difference (width $-d$ ) increases. This is due to the fact that the multiplier $(2-\sqrt{3})$ is the least as compared to $(2-\sqrt{2})$ and 1 , hence in a regular tessellable polygon the number of sides increases with a decrease in the overlap difference. Thus, as $n \rightarrow \infty$ for regular tessellable polygon, then $d \rightarrow 0$. Our study also reveals that the area of a non-tessellable regular polygon inscribed in a circle can be calculated using the formula $A_{n}=\frac{n R_{1}^{2}}{2} \sin \left(\frac{2 \pi}{n}\right)$ where $n$ is the number of sides and $R_{1}$ as the radius of the circle.

\section{Discussion}

Geometry of tessellable regular polygon resulted in hexagonal area of $\frac{3 \sqrt{3}}{2} R_{1}^{2}$ which approximates closely the area of circle than any other tessellable regular polygon for disk covering. This is a $17.3 \%$ reduction over the disks area of $\pi R_{1}^{2}$. L' Hopital's rule was used to established the fact that the area of regular polygon inscribed in a disk limit to the area of a circle as $\mathrm{n}$ increases. Hexagon has a segment overlap of $8.655 \%$ compared to $18.175 \%$ for square and $29.33 \%$ for equi-triangular tilling in disk covering. Hence hexagon has the least overlap area therefore with least material cost for disk covering. Hexagonal tiling as a guide to disk covering is proved to have the least overlap difference of $(2-\sqrt{3}) R_{1}$ which is $13.4 \%$ over the diameter of the disk. This implies that regular hexagon has the minimum width and therefore is the best geometric object for optimal disk covering in a plane A formulae for apothem $r_{n}=R_{1}$ os $\left(\frac{\pi}{n}\right)$ and total overlap difference $d_{n}=2 n R_{1}\left[1-\cos \left(\frac{\pi}{n}\right)\right]$ for tessellable regular polygon inscribed in disks for covering were put forward.

That of the area was found to be $\left[\pi-n \sin \left(\frac{2 \pi}{n}\right)\right] R_{1}^{2}$.

\section{Conclusions and Recommendations}

The findings in this study suggest that disks covering using hexagonal tessellation offers an optimal covering area of 82.7\% per disks area. We use both geometry and analytical approach to establish the fact that the area of a regular polygon approximates the area of a circle as the number of sides increases. The study also shows a formulae for computing the overlap difference and the apothem of tessellable regular polygon inscribed in disks for covering. We establish formulae for computing the total overlap area for regular tessellable polygon and it is the first study to propound these formulae as well as use both geometry and analysis to establish approximation of regular polygon to that of a circle. Geometric disk covering which is an important study in computational geometry, geometric topology (rubber sheet geometry) as well as optimization of telecommunication network design can best be achieved in least time complexity using hexagonal tessellation.. Therefore, Pure and applied Mathematicians, Computer Scientists as well as Telecommunication engineers should not lose sight of this important finding when covering with disks.

\section{References}

Ding, R. (2010). On Counting Problems in Archimedean Tiling's- A Survey. Bernoulli Conference on Discrete and Computational Geometry. Hebei Normal University, pp. 7. Access: http://dcgprogram.epfl.ch/files/content/sites/dcgprogram/files/users/184244/public/ren_ding.pdf

Grunbaum, B., \& Shepard, G. S. (1986). Tiling and Patterns, Freeman, New-York. Access: http://dcgprogram.epfl.ch/files/content/sites/dcgprogram/files/users/184244/public/ren_ding.pdf

$\mathrm{Hu}$, N. (2013). Approximation Algorithms for Geometric Covering Problems for Disks and Squares. Masters Thesis, University of Waterloo, Ontario, Canada. Access: https://uwspace.uwaterloo.ca/bitstream/handle/10012/7703/Hu_Nan.pdf?sequence=1

Keating, K., \& King, J. (1999). Signed Tiling's with Squares. Journal of Combinatorial Theory - Series A, 85(1), 83-91. http://dx.doi.org/10.1006/jcta.1998.2902. 
Lessard, D. (2000). Optimal Polygon Placement on a Grid. Masters Thesis, Ottawa-Carleton Institute for Computer Science, Carleton University, Canada, pp. 17. Access: http://www.collectionscanada.gc.ca/obj/s4/f2/dsk1/tape3/PQDD_0028/MQ52380.pdf

Melissen, J., \& Schuur, P. (1996). Improved coverings of a square with six and eight equal circles. Electronic Journal of Combinatorics, 3(1). http://dx.doi.org/10.1016/S0166-218X(99)00130-4.

Paredes, R., Aragon, J. L., \& Barrio, A. (1998). Non-periodic Hexagonal Square-Triangle Tiling's. Physical Review B, 58(18), 11990-11995. http://dx.doi.org/10.1103/PhysRevB.58.11990

Raposo P. (2011). Scale-Specific Automated map Line Simplification by Vertex Clustering on A Hexagonal Tessellation, Masters Thesis, Pennsylvania State University, USA. pp.37.

Sirbu A. (1992). Some Tiling's Problems. Tensor, 54, pp.174-178.

Xu, X., \& Whang, Z. (2011). Wireless Coverage Via Dynamic Programming. Illinois Institute of Technology, Chicago, USA. WASA, LNCS 6843, pp. 108 - 118. http://dx.doi.org/10.1007/978-3-642-23490-3_10

\section{Copyrights}

Copyright for this article is retained by the author(s), with first publication rights granted to the journal.

This is an open-access article distributed under the terms and conditions of the Creative Commons Attribution license (http://creativecommons.org/licenses/by/3.0/). 